\title{
Analysis of risk factors for revision in distal femoral fractures treated with lateral locking plate: a retrospective study in Chinese patients
}

\author{
Guojin Hou, Fang Zhou* (D), Yun Tian, Hongquan Ji, Zhishan Zhang, Yan Guo, Yang Lv, Zhongwei Yang and
} Yawen Zhang

\begin{abstract}
Background: To analyze the risk factors of revision operation after the treatment of distal femoral fracture with lateral locking plate (LLP).

Methods: Retrospective analysis of the clinical data of 152 cases with distal femoral fracture treated in our hospital from March 2005 to March 2019. The SPSS 26.0 software (univariate analysis and logistic regression analysis) was used to analyze the general condition, fracture-related factors, operation-related factors, and construct characteristics of internal fixation.

Results: Sixteen of 152 patients who were included in the study underwent revision surgery, with a revision rate 10.5\%. Univariate analysis showed that there were significant differences in age, body mass index (BMI), fracture type, supracondylar involved or not, type of incision, quality of reduction, ratio of length of plate/fracture area (R1), the ratio of the length of the plate/fracture area above the condylar (R2), ratio of distance between proximal part of fracture and screw/ working length of proximal plate (R3) between the two groups $(P<0.05)$. Logistic regression analysis showed that age [OR for age $>61.5$ group is $4.900(1.071-22.414)$ ], fracture type [OR for A3 fracture is $8.572(1.606-45.750)$, the OR for periprosthetic fracture after TKA is $9.073(1.220-67.506)]$, poor reduction quality [OR is $7.663(1.821-32.253)]$, and the ratio of the length of the plate/fracture area above the condylar were the possible risk factors $(P<0.05)$.

Conclusion: Age, fracture type (A3 and periprosthetic fracture after TKA), poor reduction quality, and the ratio of the length of the plate/fracture area above the condylar were the possible risk factors of the revision in distal femoral fractures treated with lateral locking plate. The appropriate application of the locking plate and operation strategy are the key to reduce the revision rate in distal femoral fractures.
\end{abstract}

Keywords: Distal femoral fracture, Periprosthetic fracture, Total knee arthroplasty, Locking plate, Lateral, Revision, Risk factors

\section{Background}

The incidence of distal femoral fracture accounting for 4$6 \%$ of femoral fractures [1]. The distribution of patient' $s$ age is bimodal, the younger patients are mostly caused by high energy injury, while the older patients are mostly

\footnotetext{
* Correspondence: zhouf@bjmu.edu.cn

Department of Orthopaedic Surgery, Peking University Third Hospital, No 49, North Garden Road, HaiDian District, Beijing 100191, China
}

combined with osteoporosis and low energy mechanism such as falls from standing height. For both groups, surgical treatment of distal femoral fracture should fully consider many factors, such as the patient's physical condition, bone stock, pattern and position of fracture, articular surface involvement, comminution degree, and the presence of an adjacent implant. At present, there are many kinds of internal fixators available, such as $95^{\circ}$ angle plate, dynamic

(c) The Author(s). 2020 Open Access This article is licensed under a Creative Commons Attribution 4.0 International License, which permits use, sharing, adaptation, distribution and reproduction in any medium or format, as long as you give appropriate credit to the original author(s) and the source, provide a link to the Creative Commons licence, and indicate if changes were made. The images or other third party material in this article are included in the article's Creative Commons licence, unless indicated otherwise in a credit line to the material. If material is not included in the article's Creative Commons licence and your intended use is not permitted by statutory regulation or exceeds the permitted use, you will need to obtain permission directly from the copyright holder. To view a copy of this licence, visit http://creativecommons.org/licenses/by/4.0/ The Creative Commons Public Domain Dedication waiver (http://creativecommons.org/publicdomain/zero/1.0/) applies to the data made available in this article, unless otherwise stated in a credit line to the data. 
condyle plate, lateral locking plate (LLP) of distal femur, and retrograde intramedullary nail. LLP has become increasingly popular since the technique was introduced in the late 1990s for its minimally invasive implantation, less soft tissue/blood supply destruction, and advantages of angle stability [2, 3]. However, with the accumulation of cases, initial success rates of the treatment of distal femoral fractures with LLP have given way to high incidence of complications $32 \%$, such as delayed union, nonunion, and failure of internal fixation, among which the incidence of nonunion could be as high as $0-21 \%[4,5]$. This increase may be multifactorial and attributable to an increased use of the technique, which is an application to a broader range of patient types. Distal femoral nonunions are disastrous and associated with axial malalignment, chronic pain, loss of ambulatory function, and decreased knee range of motion (ROM) [6].

The purpose of this study was to retrospectively analyze the clinical date of patients admitted to our hospital and to identify patient characteristics, injury, first operation, and construct characteristics that are independent predictors of increased risk of revision when LLP is used to treat distal femoral fractures. Using this data, a model was built to predict which patients admitted with distal femoral fracture would need revision. Measures to promote healing such as medical intervention, early bone grafting, and medial plate addition may be implemented when high-risk cases were identified. By better managing this process, patients may receive optimal medical care without catastrophic complications.

\section{Materials and methods}

\section{Patient data}

This retrospective study was based on data gathered from the hospital electronic medical record (EMR) system and the blood bank database. Approval was taken from the local research committees. Clinical data of the patients with distal femoral fracture treated in our hospital from March 2005 to March 2019 were analyzed. The revision was defined as the need for reoperation due to nonunion or failure of internal fixation.

Inclusion criteria were the following: fresh fracture $(<$ 3 weeks), age $>18$ years old, treated with LLP, and follow-up data before fracture healing. Exclusion criteria were as follows: old fracture ( $>3$ weeks), age $<18$ years old, pathological fracture, AO/OTA type 33-B fracture, and no follow-up data.

The clinical data of evaluation include the following: (1) patient characteristics: gender, age, body mass Index (BMI), comorbidity (diabetes mellitus, steroids use), tobacco, and alcohol addiction; (2) injury-related factors: injury cause, open or closed injury, fracture AO/OTA classification, and supracondylar area involvement; (3) operation-related factors: incision, operation time, and reduction quality; (4) construction of fixation (Fig. 1): length of plate L1/length of fracture area L2 (R1), the length of the plate above the condylar screw L3/the length of the fracture area L2 (R2), density of the proximal condylar screw (D) (the number of screws placed above the proximal condylar screw/the holes), and the distance between proximal part of fracture and screw L4/the working length of the proximal plate L5 (R3).

\section{Surgical technique and postoperative treatment}

All procedures were performed by the senior surgeons and use general or intraspinal anesthesia, supine position, with ilium pillow placed under the hip of the affected side. The lateral approach of para-knee joint or the para-patella was used with a bolster in the supracondylar region. A medial minimally invasive incision was performed to assist reduction according to the reduction. It is important to restore axial alignment, length, and rotation. All patients were fixed with LLP (Synthes, USA). No autogenous iliac bone transplantation was performed in the first operation.

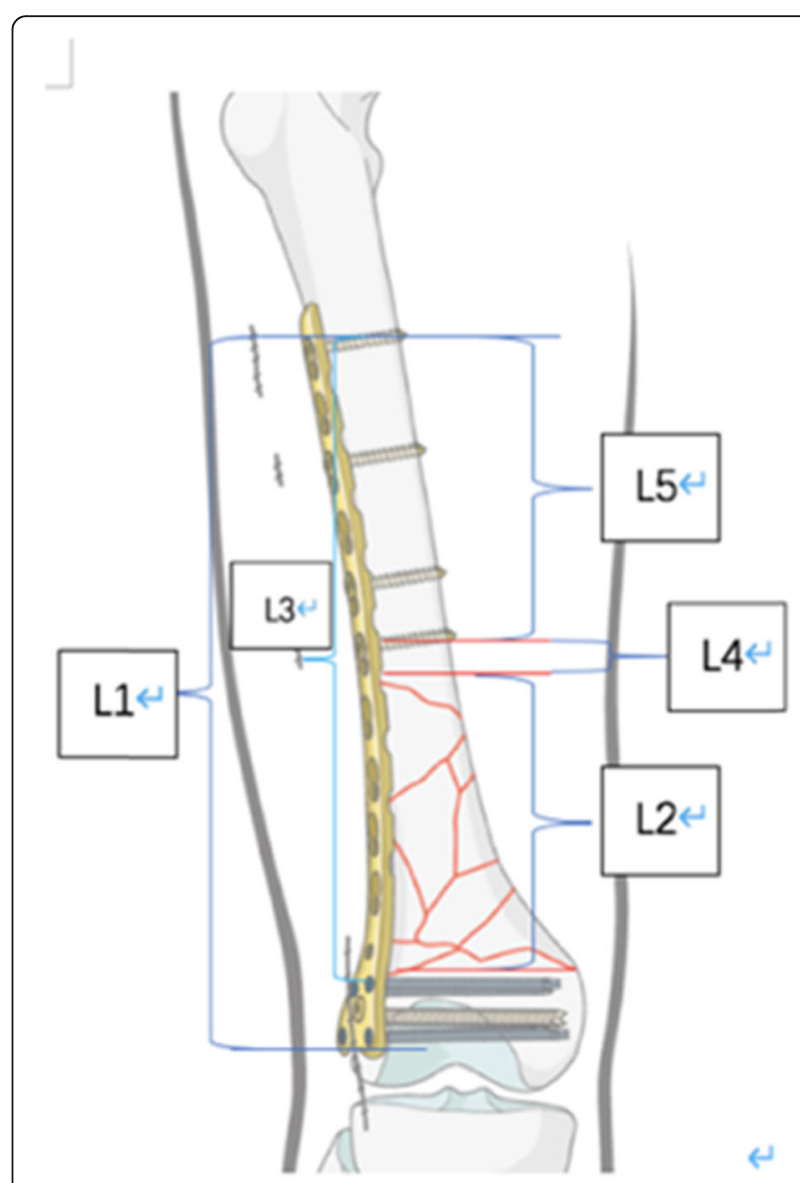

Fig. 1 Internal fixation structure. L1, length of plate; L2, length of fracture area; $L 3$, length of plate above condylar screw; $L 4$, distance between proximal part of fracture and screw; $L 5$, working length of proximal plate 
The patients did not need external fixation after operation if the fracture was satisfactorily fixed. Isometric contraction exercise of quadriceps femoris and ankle pump exercise began after recovery of anesthesia. Rehabilitation exercise was carried out after 2 weeks; the injured limb was not loaded within 8 weeks, and the load was gradually increased according to the fracture healing. The X-ray of knee and femur was reexamined at 1 , 3,6 , and 12 months after operation and regularly reexamined every 2 months until the fracture healed for delay union patients.

\section{Statistical analysis}

Statistical analysis was conducted with the SPSS 26.0 software (SPSS Inc., USA). Univariate analysis was used to compare the group which received revision and the group that did not. Continuous variables that follow a normal distribution were analyzed using a two-sample Student $t$ test; continuous variables that follow a nonnormal distribution were analyzed using the Mann Whitney $U$ test; Pearson chi-square test and Fisher's exact test were used to compare the groups with respect to categorical variables. A $P$ value $<0.05$ was considered to indicate statistical significance.

A logistic regression was applied to identify the significant independent predictors for revision. The full regression model included risk factor candidates based on univariate analysis. Model selection methods such as Wald-backward elimination were used in order to identify important factors from the explanatory variables.

\section{Results}

A total of 152 acute distal femur fracture patients met inclusion criteria. Of these, 16 fractures were surgically revised for nonunion, with a revision rate $10.5 \%$. Patients were divided into non-revision group and revision group. Median follow-up for all 152 fractures was 20 months (range, 9-168 months). Demographic data and the univariate analysis of patient, fracture, first operation, and construct characteristics associated with revision were summarized in Table 1.

Risk factors for revision in distal femoral fractures treated with LLP were assessed with univariate analysis (Table 1). Significant different factors $(P<0.05)$ were as follows: age, $\mathrm{BMI}$, fracture type, supracondylar involvement, type of incision, quality of reduction, R1, R2, and R3.

Logistic regression was performed in order to simulate a decision analysis. Four out of 9 independent variables were found to have a statistically significant effect on the rate of revision in distal femoral fractures treated with lateral locking plate: age, fracture type, reduction quality, and the ratio of the length of the plate/fracture area above the condylar. Regression coefficients, likelihood ratios, $p$ values, adjusted odds ratios, and 95\% confidence intervals were determined (Table 2).

A receiver operating characteristic (ROC) curve analysis was used to evaluate the predictive performance of the logistic regression model and its ability to predict the rate of revision after in distal femoral fractures treated with lateral locking plate (Fig. 2). The area under the curve was 0.877, which demonstrated a good diagnostic performance. ROC curve analysis was also used to evaluate age and $\mathrm{R} 2$ and its ability to predict the rate of revision (Fig. 2). Age $=61.5, \mathrm{R} 2=1.89$, and predicted probability (Fig. 3) $P$ (revision $)=0.059$ was selected as an optimal cutoff point that best differentiates between patients who should receive revision and those who should not. This cutoff point has the highest sensitivity and specificity rates.

\section{Discussion}

Compared with the traditional angle plate and dynamic condylar plate, the LLP is the most commonly used method nowadays for the treatment of distal femoral fracture for its advantages of minimally invasive, less soft tissue interference, and angular stability [7, 8]. However, there are more and more reports about the complications of LLP in the treatment of distal femoral fracture in recent years [9]. Nonunion of distal femoral fracture is a disastrous complication and seriously affects the quality of life of patients for decreased joint activity and pain. This study attempts to analyze the general situation of patients, fracture-related factors, operation, and construct characteristics to explore the risk factors of revision of LLP in the treatment of distal femoral fracture. The importance of this study is to identify high-risk patients and conduct interventions to promote healing as early as possible, which may reduce the rate of revision in future treatment.

Old age is related to the occurrence and degree of osteoporosis. The probability of internal fixation loosening and fracture nonunion increases in serious osteoporosis patients for the lower holding power of screws [10]. This study suggested similar results; the average age of the non-revision and revision group were $61.6 \pm 14.7$ years and $69.0 \pm 10.0$ years respectively; the OR for revision in age > 61.5 group is 4.900 (1.071-22.414).

The principle of "tension band" was used in the treatment of distal femoral fracture with LLP, and the integrity of medial cortex is important and should be restored. $\mathrm{AO} /$ OTA type A3 comminuted fracture can cause comminution of medial cortex of metaphysis and destroy its medial supporting ability. In this condition, the tension on the LLP will become repeated bending stress, which can lead to fatigue of the plate; even plate failure or screw loosening, the OR for revision in AO/OTA type A3 group is $8.572(1.606-45.750)$, which is consistent with the previous literature [11, 12]. For this reason, some scholars 
Table 1 General characteristics and univariate analysis of risk factors for revision after in distal femoral fractures treated with lateral locking plate

\begin{tabular}{|c|c|c|c|c|}
\hline Variable & Non revision group & Revision group & $t / z / x^{2}$ & $P$ \\
\hline Number & 136 & 16 & - & - \\
\hline Gender (male/female) & $28 / 108$ & $4 / 12$ & 0.168 & $0.682^{*}$ \\
\hline Age (years) & $61.6 \pm 14.7$ & $69.0 \pm 10.0$ & -2.645 & $0.014^{*}$ \\
\hline DM (yes/no) & $36 / 100$ & $6 / 10$ & 0.871 & $0.351^{\ddagger}$ \\
\hline Tobacco/alcohol (yes/no) & $8 / 128$ & $2 / 14$ & - & $0.284^{*}$ \\
\hline Steroid usage & $6 / 130$ & $0 / 16$ & - & $0.507^{*}$ \\
\hline BMI & $25.4 \pm 3.8$ & $27.3 \pm 2.1$ & -3.005 & $0.006^{*}$ \\
\hline Reason of injury (high/low energy) & $80 / 56$ & $10 / 6$ & 0.080 & $0.777^{£}$ \\
\hline Open/closed & $12 / 124$ & $0 / 16$ & - & $0.366^{*}$ \\
\hline Fracture type (A2/A3/C1/C2/PF) & $24 / 42 / 10 / 42 / 18$ & $0 / 10 / 0 / 2 / 4$ & 11.223 & $0.024^{ \pm}$ \\
\hline Supracondylar involved (no/yes) & $70 / 66$ & $4 / 12$ & 4.015 & $0.045^{f}$ \\
\hline Incision (lateral/lateral + medial) & $106 / 30$ & $8 / 8$ & 5.961 & $0.015^{f}$ \\
\hline Duration of operation (minutes) & $144.2 \pm 45.9$ & $163.4 \pm 55.0$ & -1.550 & $0.123^{*}$ \\
\hline Quality of reduction (good/bad) & $96 / 40$ & $5 / 11$ & 9.937 & $0.002^{f}$ \\
\hline R1 & $3.17 \pm 1.43$ & $2.54 \pm 0.67$ & 2.997 & $0.005^{*}$ \\
\hline R2 & $3.31 \pm 1.32$ & $2.45 \pm 0.72$ & 3.970 & $0.000^{*}$ \\
\hline R3 & $0.35 \pm 0.29$ & $0.19 \pm 0.18$ & 2.094 & $0.038^{*}$ \\
\hline Density of supracondylar screws & $0.59 \pm 0.15$ & $0.65 \pm 0.18$ & -1.590 & $0.114^{\#}$ \\
\hline
\end{tabular}

$D M$ diabetes mellitus, $B M I$ body mass index, $P F$ periprosthetic fracture after total knee arthroplasty, $R 1$ ratio of length of plate/fracture area, $R 2$ the ratio of the length of the plate/fracture area above the condylar, $R 3$ ratio of distance between proximal part of fracture and screw/working length of proximal plate *Fisher's exact test

${ }^{*}$ Two-sample Student $t$ test

${ }^{\mathrm{E}}$ Chi-square test

"Mann Whitney $U$ test

The values are given as the mean and the standard deviation for continuous variables and as the number of patients for categorical variables

suggest double plate fixation for A3 and C3 type comminuted fractures to improve the fracture healing rate [13].

Periprosthetic fracture after total knee arthroplasty (TKA) is a special type of distal femoral fracture. Although single LLP has less degree of soft tissue damage and certain angle fixation stability, it still may not provide enough stability in this certain condition. In order to overcome these problems, Kim et al. [14] used double plate technique to provide enough stability and reduce the damage of soft tissue as much as possible. This method is especially suitable for distal femoral fracture patients after TKA with poor bone stock, comminuted fracture, and far periprosthetic fracture line [13]. Also, double plated construct had greater stabilization in a simulated fracture model when compared to a single lateral plate [15]. If it is unable to maintain satisfactory alignment and sufficient stability, double plate should be used for fixation in periprosthetic fractures after TKA.

Reduction is the basic AO principle for the treatment of fractures. Poor reduction of distal femoral fractures and residual gap at the fracture end can cause excessive local interfragmentary movement and bone absorption

Table 2 Logistic regression model for predicting revision in patients with distal femoral fractures

\begin{tabular}{|c|c|c|c|c|c|c|}
\hline Predictors & Regression coefficient & Standard error & Wald $x^{2}$ & $P$ value & OR & OR 95\% Cl \\
\hline Age $(X 1)$ & 0.096 & 0.036 & 7.130 & 0.008 & 1.100 & $1.026-1.180$ \\
\hline Type of fracture (X2) & & & 6.750 & 0.034 & & \\
\hline Type of fracture (A3) & 2.419 & 0.854 & 6.323 & 0.012 & 8.572 & $1.606-45.750$ \\
\hline Type of fracture (PF) & 2.205 & 1.024 & 4.639 & 0.031 & 9.073 & $1.220-67.506$ \\
\hline Quality of reduction (X3) & 2.036 & 0.733 & 7.713 & 0.005 & 7.663 & $1.821-32.253$ \\
\hline R2 (X4) & -1.127 & 0.425 & 7.011 & 0.008 & 0.324 & $0.141-0.746$ \\
\hline Constant & -7.879 & 2.509 & 9.861 & 0.002 & 0.000 & - \\
\hline
\end{tabular}

$P F$ periprosthetic fracture after total knee arthroplasty, $R 2$ ratio of the length of the plate/fracture area above the condylar

The classification of each factor: fracture type $\mathrm{X} 2: 1=\mathrm{A} 2 / \mathrm{C} 1 / \mathrm{C} 2$ fracture, $2=\mathrm{A} 3$ fracture, $3=\mathrm{PF} ; \mathrm{X} 3: 1=$ satisfactory reduction, $2=$ poor reduction 


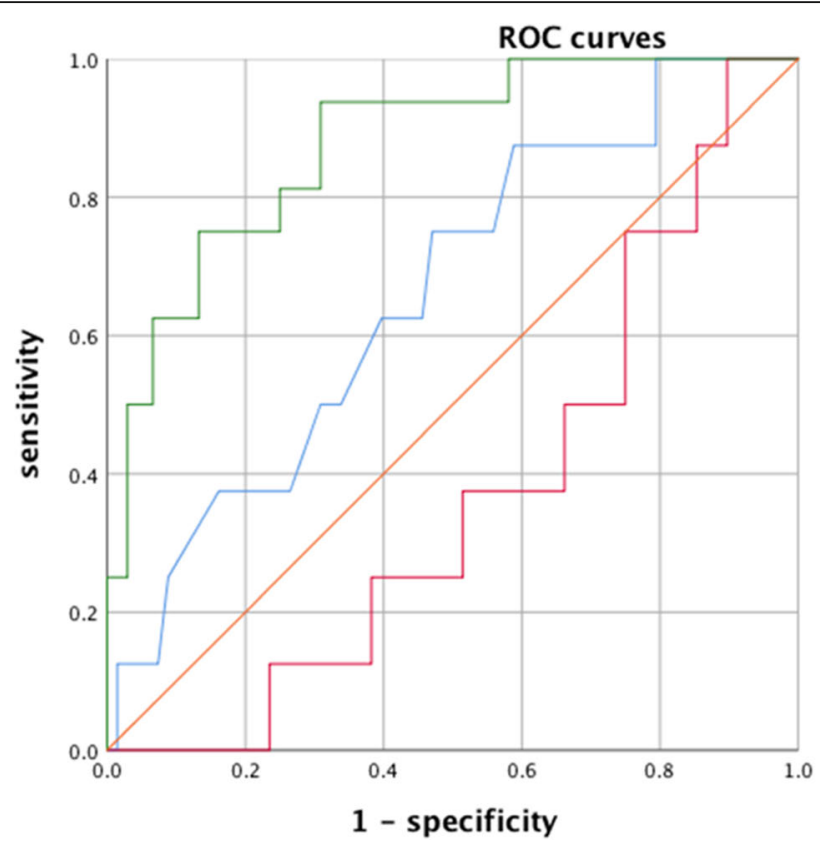

\section{Source of curves}

- age

- R2

- predicted probability

- Reference line

Fig. 2 ROC curve analyses were used to evaluate the predictive performance of logistic regression model, age, and R2 to predict revision

[16]. Also, it cannot restore the support ability of the medial cortex and increase the bending stress of the LLP, which may accelerate fatigue of the plate. Pesciera et al. [17] pointed out that the rate of nonunion could be as high as $12 \%$ when the medial alignment was poor and the medial defect was greater than $2 \mathrm{~cm}$. After the concept of biological osteosynthesis (BO) was introduced, protection of soft tissue blood supply at fracture end was widely valued. Therefore, we should restore axial alignment, length, and rotation and try our best to reduce the damage to soft tissue.

Because of the special design of the LLP of the distal femur, its distal shape and the number of screws inserted are constant; therefore, the author thought that the "real" working length of the plate should be considered and introduced the concept of R2: ratio of the length of the plate/fracture area above the condylar. Logistic regression analysis shows that it is a risk factor for revision. Tan et al. [18] pointed out that the insufficient length of the plate may be the risk factor of internal fixation failure for it cannot disperse the stress effectively. The weakest part of LLP is the dynamic hole around the fracture; when the plate concentrates too much stress on a short distance, this part can break out. If there is osteoporosis at the same time, screw loosening and pulling out are more common [19]. In addition, Elkins et al. [20] pointed out that the possible causes of distal femoral fracture nonunion also include too strong LLP structure as to inhibit the movement of fracture end. Therefore, in addition to recent technologies and advances in the management of distal femoral fractures, surgeon needs to always bear in mind the basic principles ruling the plating fixation of distal femur, i.e., apply a sufficiently long plate, maximize the screw fixation to the distal part, and avoid over-rigid fixation [12, 21].

There are still some deficiencies in this study: retrospective study, the sample size is small; there is no analysis of the impact of different surgeon; this paper does not analyze the impact of other potential confounding factors on fracture healing; further prospective randomized controlled clinical trials are needed to verify the results.

\section{Conclusion}

Lateral locking plate is one of the effective internal fixation options in the treatment of distal femoral fracture, but the incidence of complications is not low. Age, fracture type (A3 and periprosthetic fracture after TKA), poor reduction quality, and the ratio of the length of the plate/fracture area above the condylar are the predictive

$$
P(\text { revision })=\frac{1}{\left.1+\exp ^{-(7.879+0.096 * a g e}+2.419 * \mathrm{~A} 3+2.205 * \mathrm{PF}+2.036 * \mathrm{Q}-1.127 * \mathrm{R} 2\right)}
$$

Fig. 3 A predictive formula based on the significant risk factors model used to predict the need for revision. PF, periprosthetic fracture after total knee arthroplasty; Q, quality of reduction; R2, ratio of the length of the plate/fracture area above the condylar 
factors for revision after the treatment of distal femoral fracture with LLP. We should choose the appropriate plate and operation strategy according to the type of fracture and patient characteristics to reduce the revision rate. When high-risk cases were identified, interventions should be conducted to promote healing as early as possible and improve the prognosis.

\section{Abbreviations}

LLP: Lateral locking plate; ROM: Range of motion; EMR: Electronic medical record; BMI: Body mass index; DM: Diabetes mellitus; Cl: Confidence intervals; ROC: Receiver operating characteristic; PF: Periprosthetic fracture after total knee arthroplasty; TKA: Total knee arthroplasty; BO: Biological osteosynthesis

\section{Acknowledgements}

The authors would like to thank Xiaoyan Niu, the secretary, for a lot of image preparation and data transmission work, which ensured the smooth progress of the project.

\section{Authors' contributions}

GJH were involved in designing the study, acquisition of data, and drafting the manuscript. FZ and $Y T$ designed the study, revised the manuscript critically, and gave some important suggestions; HQJ, ZSZ, YG, and YL performed the surgeries and revised the manuscript; ZWY and YWZ completed the follow-up and collected the data. All authors read and approve the final manuscript.

\section{Funding}

There is no funding source.

\section{Availability of data and materials}

The datasets used and/or analyzed during the current study are available from the corresponding author on reasonable request.

\section{Ethics approval and consent to participate}

This retrospective study was approved by the Institutional Ethics Board of The Peking university Third hospital. All enrolled patients were informed and agreed to provide relevant data for this study. The methods were carried out in accordance with the relevant guidelines.

\section{Consent for publication}

Not applicable.

\section{Competing interests}

The authors declare that they have no competing interests.

Received: 22 March 2020 Accepted: 30 July 2020

Published online: 12 August 2020

\section{References}

1. Martinet $\mathrm{O}$, Cordey J, Harder $\mathrm{Y}$, et al. The epidemiology of fractures of the distal femur. Injury. 2000;31 Suppl 3(Suppl 3):C62-3.

2. Hoffmann MF, Jones CB, Sietsema DL, et al. Clinical outcomes of locked plating of distal femoral fractures in a retrospective cohort. J Orthop Surg Res. 2013:8(1):43.

3. Consortium S F. LCP versus LISS in the treatment of open and closed distal femur fractures: does it make a difference? J Orthop Trauma. 2016;30(6): e212-6.

4. Henderson CE, Kuhl LL, Fitzpatrick DC, et al. Locking plates for distal femur fractures: is there a problem with fracture healing? J Orthop Trauma. 2011 25 Suppl 1(2):S8-14.

5. Rodriguez EK, Boulton C, Weaver MJ, et al. Predictive factors of distal femoral fracture nonunion after lateral locked plating: a retrospective multicenter case-control study of 283 fractures. Injury. 2014;45(3):554-9.

6. Gardner MJ, Toro-Arbelaez JB, Harrison M, et al. Open reduction and internal fixation of distal femoral nonunions: long-term functional outcomes following a treatment protocol. J Trauma. 2008;64:434-8.
7. Syed AA, Agarwal M, Giannoudis PV, et al. Distal femoral fractures: longterm outcome following stabilisation with the LISS. Injury. 2004;35(6):599607.

8. Kregor PJ, Stannard JA, Zlowodzki M, et al. Treatment of distal femur fractures using the less invasive stabilization system. J Orthop Trauma. 18(8): 509-20.

9. Lujan T, Henderson CE, Madey SM, et al. Locked plating of distal femur fractures leads to inconsistent and asymmetric callus formation. J Orthop Trauma. 2010;24(3):156-62.

10. Hak DJ, Toker $S, Y i$, et al. The influence of fracture fixation biochemistry on fracture healing. Orthopedias. 2010;33:752-5.

11. Fulkerson E, Egol KA, Kubiak EN, et al. Fixation of diaphyseal fractures with a segmental defect: a biochemical comparison of locked and conventional placement techniques. Trauma. 2006;60:830-5.

12. Ricci WM, Streubel PN, Morshed S, et al. Risk factors for failure of locked plate fixation of distal femur fractures: an analysis of 335 cases. J Orthop Trauma. 2014;28:83-9.

13. Steinberg EL, Elis J, Steinberg Y, et al. A double playing approach to total femur fracture: a clinical study. Injury. 2017:48(10):2260-5.

14. Kim W, Song JH, Kim JJ. Periprosthetic fractures of the distal femur following total knee arthroplasty: even very distal fractures can be successfully treated using internal fixation. Int Orthop. 2015:39(10):1951-7.

15. Muizelaar A, Winemaker MJ, Quenneviile CE, et al. Preliminary testing of a novel bilateral plating technique for treating periprosthetic fractures of the distal femur. Clin Biomech. 2015;30(9):921-6.

16. Jagodzinski $M$, Krettek $C$. Effect of mechanical stability on fracture healing-an update. Injury. 2007;38(1-supp-S):S3-10.

17. Pesciera V, Staletti L, Cavanna M, et al. Predicting the failure in fatal femur fractures. Injury. 2018;49(11):suppl 3: s2-7.

18. Tan SL, Balogh ZJ. Indications and limitations of locked playing. Injury. 2009; 40:683-91.

19. Peng N, Ming Z, Yuedong Z. Logistic regression analysis of failure of locking compression plate internal fixation for lower limb fracture. Chin J Orthoped. 2014;22(24):2238-43

20. Elkins J, Marsh JL, Lujan T, et al. Motion predicts clinical callus formation: construct-specific finite element analysis of supracondylar femoral fractures. J Bone Joint Surg Am. 2016:98(4):276-84.

21. Ricci WM. Periprosthetic femur fractures. J Orthop Trauma. 2015:29:130-7.

\section{Publisher's Note}

Springer Nature remains neutral with regard to jurisdictional claims in published maps and institutional affiliations.

\section{Ready to submit your research? Choose BMC and benefit from:}

- fast, convenient online submission

- thorough peer review by experienced researchers in your field

- rapid publication on acceptance

- support for research data, including large and complex data types

- gold Open Access which fosters wider collaboration and increased citations

- maximum visibility for your research: over $100 \mathrm{M}$ website views per year

At $\mathrm{BMC}$, research is always in progress.

Learn more biomedcentral.com/submission 